\title{
Cancellation of Unwanted Doppler Radar Sensor Motion Using Empirical Mode Decomposition
}

\author{
Isar Mostafanezhad, Member, IEEE, Ehsan Yavari, Student Member, IEEE, \\ Olga Boric-Lubecke, Senior Member, IEEE, Victor M. Lubecke, Senior Member, IEEE, \\ and Danilo P. Mandic, Fellow, IEEE
}

\begin{abstract}
The operation of microwave Doppler radar for sensing physiological motion signals is heavily compromised under sensor motion. To that end, we investigate the feasibility of applying empirical mode decomposition method in this context, and demonstrate its effectiveness in removing sensor motion artifacts. This method is shown to be effective in canceling unwanted sensor motion with precision sufficient to enable accurate heart rate extraction. Theoretical analysis and simulation results illustrate the potential of the proposed approach for a wide range of frequency separation and amplitude ratios of physiological signals and motion artifacts. Experimental results confirm that separation success is not very sensitive to amplitude ratio. A heart rate is extracted with RMSE within 1 beat per minute even in the presence of mechanical motion and order of magnitude larger in amplitude than that of the heart signal.
\end{abstract}

Index Terms-Biomedical signal detection, doppler radar, empirical mode decomposition, motion compensation.

\section{INTRODUCTION}

$\mathbf{M}$ ICROWAVE Doppler radar has shown significant promise for sensing physiological motion and subject detection [1]-[4]. Research efforts in the past decades have been focused on both reducing size and cost of the sensor [5] and overcoming challenges in wireless physiological monitoring, such as: null detection issue [3], non-linear demodulation [4], and motion artifacts [6]. The basic principle of continuous wave $(\mathrm{CW})$ Doppler radar sensor is that reflections from moving targets result in frequency shifts, while reflections from stationary clutter result only in dc offset. However, if the sensor itself undergoes unwanted motion, for example due to mounting surface vibrations or operator "hand-shake," this will introduce path length variations which phase modulate the received signal, in addition to that related to subject's motion.

Manuscript received May 31, 2012; accepted November 7, 2012. Date of publication January 9, 2013; date of current version April 5, 2013. This work was supported in part by the National Science Foundation under Contract ECS0428975, Contract ECS0702234, and Contract ECCS-0926076. The associate editor coordinating the review of this paper and approving it for publication was Dr. Kailash Thakur.

I. Mostafanezhad, E. Yavari, O. Boric-Lubecke, and V. Lubecke are with the Department of Electrical Engineering, University of Hawaii at Manoa, Honolulu, HI 96822 USA (e-mail: seyed@hawaii.edu; ehsan@hawaii.edu; olgabl@hawaii.edu; lubecke@ hawaii.edu).

D. Mandic is with the Department of Electrical and Electronic Engineering, Imperial College London, London SW7 2BT, U.K. (e-mail: d.mandic@imperial.ac.uk).

Color versions of one or more of the figures in this paper are available online at http://ieeexplore.ieee.org.

Digital Object Identifier 10.1109/JSEN.2013.2238376
Thus, the resulting signal will include components corresponding to sensor motion artifacts, which once combined with the desired signal interfere with life signs monitoring and detection.

The problem of undesired instrument motion is similar to that in still and video cameras where it causes motion blur in the capturing image. To that end, for instance, tonal correction was proposed in [7] to remove image blur caused by handshake of a cell-phone camera user. In a Doppler radar sensor system, an ultrasound beam was added to remove the undesired motion artifact component in [8], and a harmonic tag was used in [9] to isolate the desired target from other sources of motion. To further address this issue, a bi-static radar configuration using a sensor node in close proximity of a target was proposed in [10]. However, the use of sensor nodes requires more complex data collection that may not always be possible or desirable. An alternative method, proposed in [11], uses information about the undesired radar motion to remove its effects from the sensor output. For this purpose an accelerometer is placed on the radar antenna, while the recorded motion is used as a "prior knowledge" in the conditioning of the sensor output. However, as shown below, this method is effective only for relatively low amplitudes of unwanted motion. Notice that traditional methods like the PCA and ICA will not help with this problem. PCA is designed only for stationary data where the correlation matrix is coherent and represents correct signals statistics, which makes it possible to decompose the matrix into the principal and noise eigenspace. The Doppler radar data are non-stationary and therefore PCA would not produce meaningful results. The ICA framework is valid only where there are physically meaningful independent components. However, in the case of vital sign monitoring using Doppler radar the components of interest (heart rate, respiratory) and the fidgeting artifact are both correlated and their spectra overlap, which motivates us to exploit a datadriven method that operates on non-stationary data - the EMD.

In this paper we investigate the feasibility of applying Empirical Mode Decomposition (EMD) to Doppler radar signals to remove unwanted interference. EMD is a data driven multi-scale signal processing technique that decomposes the signal into a finite set of zero-mean oscillatory waveforms that may be AM/FM modulated, through a process referred to as "sifting" [12]. Since EMD is based on the local intrinsic time scales within the data, it is applicable to non-stationary 


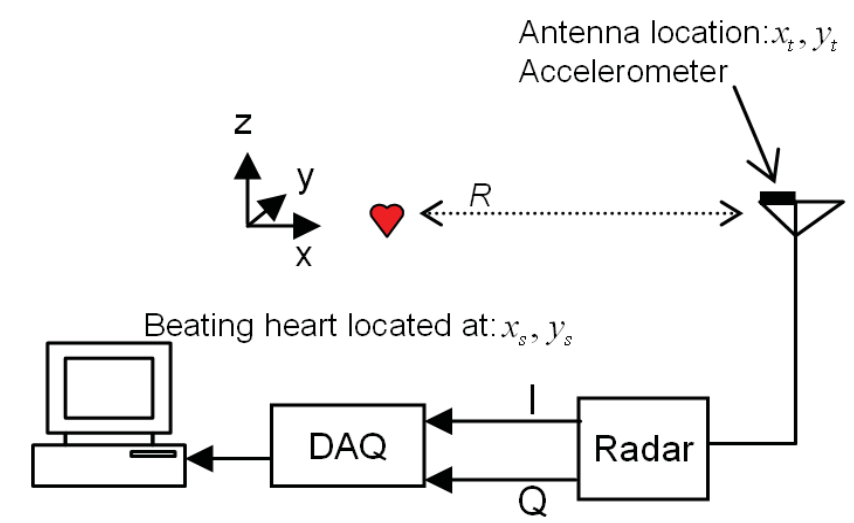

Fig. 1. Experimental setup consisting of radar sensor, data acquisition system, and computer illustrating the relative location of antenna, motion sensor (accelerometer), and the human subject's heart.

and nonlinear processes [13], and thus suitable for analyzing physiological signals in the presence of interference [14]. In [15] we have shown great promise in using EMD to remove motion artifacts due to the fidgeting of the subject. In this paper, we present the first reported experimental results that demonstrate a successful separation of the radar sensor signal components from the unwanted antenna motion and subject's physiological motion using EMD. Theoretical analysis and simulations investigate the boundaries of frequency separation and amplitude ratios for successful interference cancellation. Experimental results are consistent with the analysis and have demonstrated the feasibility of extracting heart rate with root mean square error (RMSE) of less than 1 beat per minute, even when the motion artifact amplitude is an order of magnitude larger than the magnitude of the heart signal, a situation where the method from [11] failed.

\section{Signal Model AND EMD}

Fig. 1 shows the geometry and relative positions of a radar sensor and a human subject in two dimensions.

Denote by $R$ the instantaneous radial distance of the transmitting antenna from the subject, as depicted in Fig. 1, and considering that $R$ contains both subject's motion and transmitter's unwanted shake, it can be expressed as [11]:

$$
R=R_{0}+\Delta R \approx R_{0}-a\left(x_{t}-\Delta x_{s}\right)-b\left(y_{t}-\Delta y_{s}\right),
$$

where $\Delta R$ is the distance variation due to sensor motion, while subject motion that we are interested in can be approximated as $m$ [11]:

$$
m=z-a x_{t}-b y_{t},
$$

where $z=a \Delta x_{s}+b \Delta y_{s}$ is a measure of the subject's physiological motion under observation. Parameters $a$ and $b$ are functions of geometry and position of the subject with respect to the antenna and are described in [11]. Phase demodulation of the output of the sensor approximately yields $m$. The method proposed in [11] relies on prior knowledge of antenna's motion, $x_{t}$ and $y_{t}$, to remove the effect of sensor motion artifact. For it to be effective it is important to have accurate information of the motion artifact signal. In this paper, EMD is used to decompose the demodulated

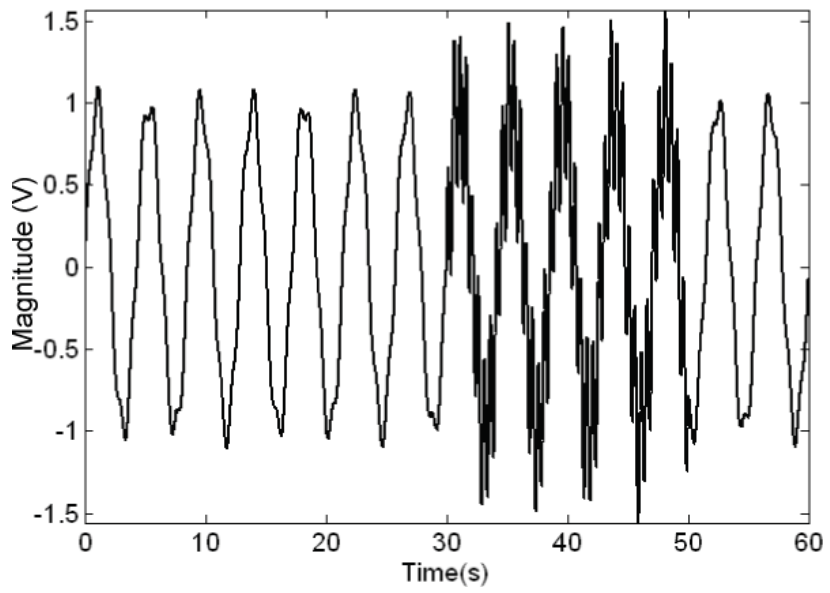

Fig. 2. Simulated radar sensor signal consisting of a respiration component, a heart component, and a motion artifact. The motion artifact component occupies the segment between $\mathrm{t}=30 \mathrm{~s}$ and $\mathrm{t}=50 \mathrm{~s}$.

sensor signal $m$ into its two components: physiological motion and motion artifact. These two signals can be considered to be AM/PM modulated components of some fundamental frequency. Physiological motion has a periodic and narrow band signature, that is, the instantaneous frequency for heart and respiration varies slowly with time [1], making EMD perfectly suited for their detection and separation [16].

Empirical Mode Decomposition (EMD) was introduced in 1998 [12] as a fully data driven technique which decomposes the signal into narrow band components called Intrinsic Mode Functions (IMFs). IMFs, denoted as $d_{k}[n]$, are functions for which 1) the number of extrema and the number of zero crossings are either equal or they differ at most by only one, and 2) the mean value of two envelopes associated with the local maxima and minima are zero. For a real-valued signal $x[n]$ the EMD is designated by the mapping:

$$
x[n]=\sum_{k=1}^{K} d_{k}[n]+r[n],
$$

where $\left\{d_{k}[n]\right\}_{k=1}^{K}$ is a set of IMFs, and $r[n]$ is the residue [12]. IMFs of the demodulated sensor signal are calculated using the sifting process, which is the basis of EMD. Once the IMFs are obtained, the motion artifact signal will be localized in an IMF; by re-synthesizing the signal through retaining only the desired components, the interference is removed from the output.

A simulation was performed in MATLAB, where respiration, heart and motion artifact signals have been superimposed to generate a simulated sensor signal, shown in Fig. 2. In this case study, the motion artifact had an amplitude two times larger than that of the heart signal and one-third of the amplitude of the respiration signal. All signals were sine waves.

The frequencies of respiration, heart, and motion artifact signals were $0.23 \mathrm{~Hz}, 1.08 \mathrm{~Hz}$, and $2 \mathrm{~Hz}$, respectively. Motion artifact was introduced between $\mathrm{t}=30 \mathrm{~s}$ and $\mathrm{t}=50 \mathrm{~s}$ and sensor output was high-pass filtered and processed using EMD (code available in [17]). High pass filtering removes the large 

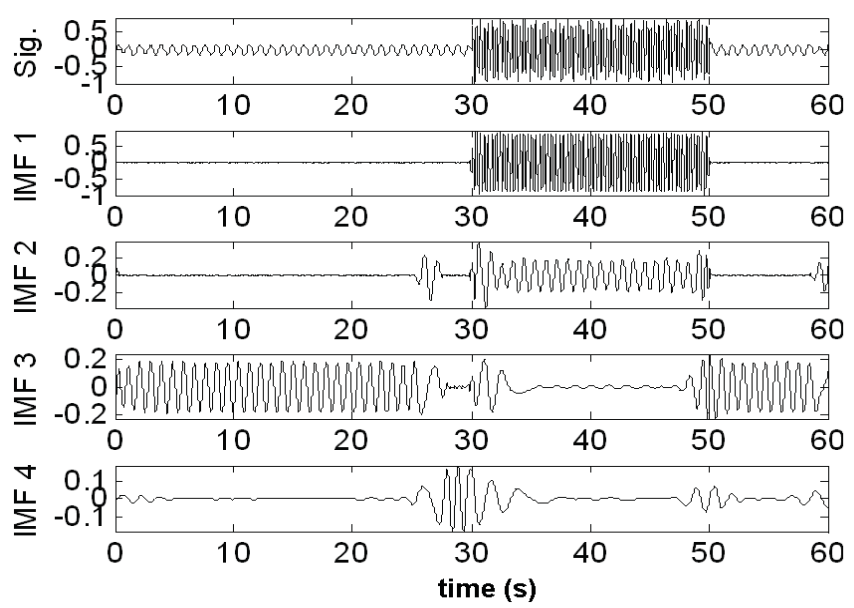

Fig. 3. High-pass filtered sensor output (top) and the IMFs within EMD.

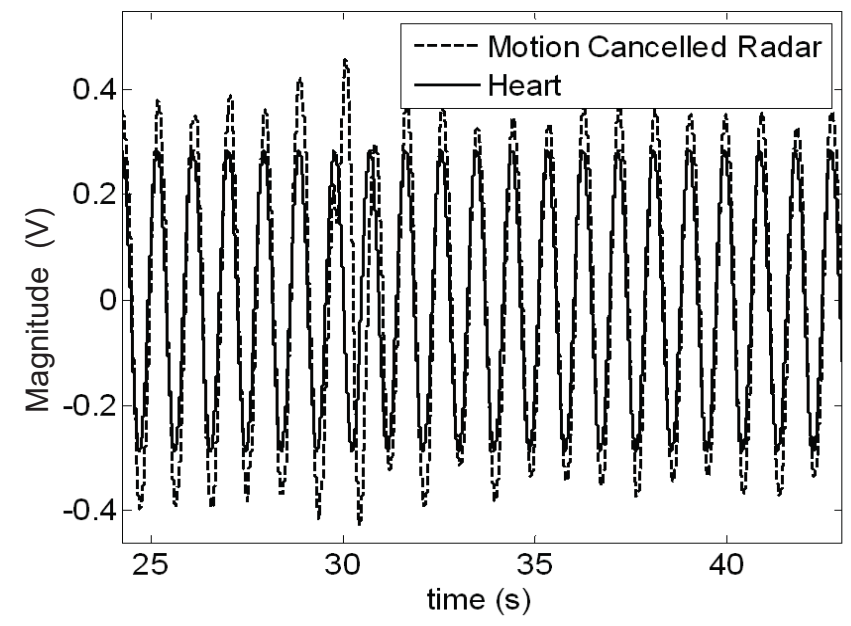

Fig. 4. Simulated motion artifact cancelled heart signal and the original heart during the presence of motion artifact. IMF2 and IMF3 were added to produce an interference-free heart signal.

respiration trace from the signal and helps the EMD algorithm generate IMFs that are related to smaller signal variations which includes heart beat components. The high pass filtered sensor output and the resulting IMFs are shown in Fig. 3.

By adding the desired IMF2 and IMF3, as identified by visual inspection, the motion artifact cancelled signal was obtained; this is shown in Fig. 4 with the original heart signal for comparison.

Since EMD is an empirical method, the results will depend on the characteristics of the signals being processed.Transients in IMF 2, IMF 3 and IMF 4 are caused by mode mixing, where one mode may leak to an adjacent mode [12], [18]. This is caused by sharp transitions in the signal, in this case at the beginning and the end of the motion artifact. However since mode mixing does not occur in the first IMF, it does not affect the final result.

In order to examine the ability of EMD to separate interference from the principal signal, a simulation was performed to investigate separation success as a function of frequency separation and amplitude ratio. We consider the problem of separating two sinusoids, signal $s$ and interference $u$ that

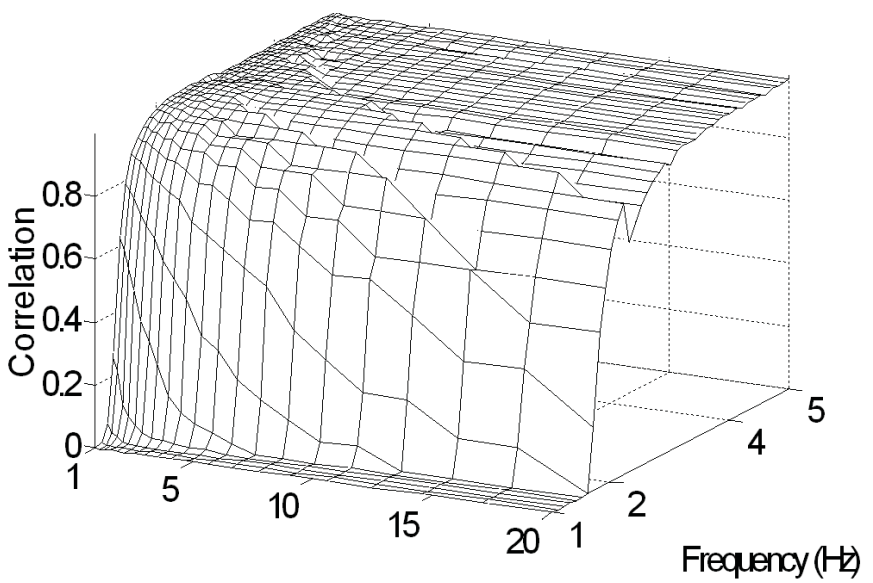

Amplitude

Fig. 5. Performance of EMD in removing interference: Resemblance (correlation) of the recovered signal to the principal signal versus interference signal's frequency and amplitude.

produce signal $x$ :

$$
x=s+u,
$$

where:

$$
\begin{aligned}
& u=a \sin (2 \pi f t) \quad a, f>1, \\
& s=\sin (2 \pi t)
\end{aligned}
$$

The goal is to recover $s$ after an interference, $u$, has been added to it. Our approach is to find the IMFs of $x$ and since frequency content of $u$ is higher than $1 \mathrm{~Hz}, u$ will appear as IMF1, while most of $s$ will be contained in IMF2.

As a measure to find how similar IMF2 and $s$ are, we use the correlation coefficient:

$$
C=\left|\frac{s \cdot I M F_{2}}{\left\|s \left|\left\|\mid I M F_{2}\right\|\right.\right.}\right|,
$$

whose values are between 0 and 1, where 0 means the two signals are not correlated and 1 that the two signals are identical. Amplitude $a$ and frequency $f$ of the interference signal are varied, and $C$ is plotted in a three-dimensional plot shown in Fig. 5. A cross section of the three-dimensional plot is shown in Fig. 6.

As an example, a measure of success in recovering the signal $s$ can be defined as a correlation of 0.5. Both Figs. 5 and 6 show that at higher interference amplitudes a larger frequency separation is required for successful separation. It can also be seen that correlation does not change much as a function of amplitude ratio for frequency separation larger than $2 \mathrm{~Hz}$. This trend is consistent with results in [18].

Fig. 6 shows the EMD performance as a function of separation frequency for interference amplitude ratios of 1 to 10. It can be observed that for equal signal and interference amplitudes, minimum separation frequency is about $1.3 \mathrm{~Hz}$. As amplitude ratio increase by an order of magnitude, minimum separation frequency for successful recovery increases by about $0.3 \mathrm{~Hz}$.

\section{EXPERIMENTAL RESULTS}

Doppler radar sensor was tested on human subjects in the presence of motion artifacts of varying amplitude. The block 


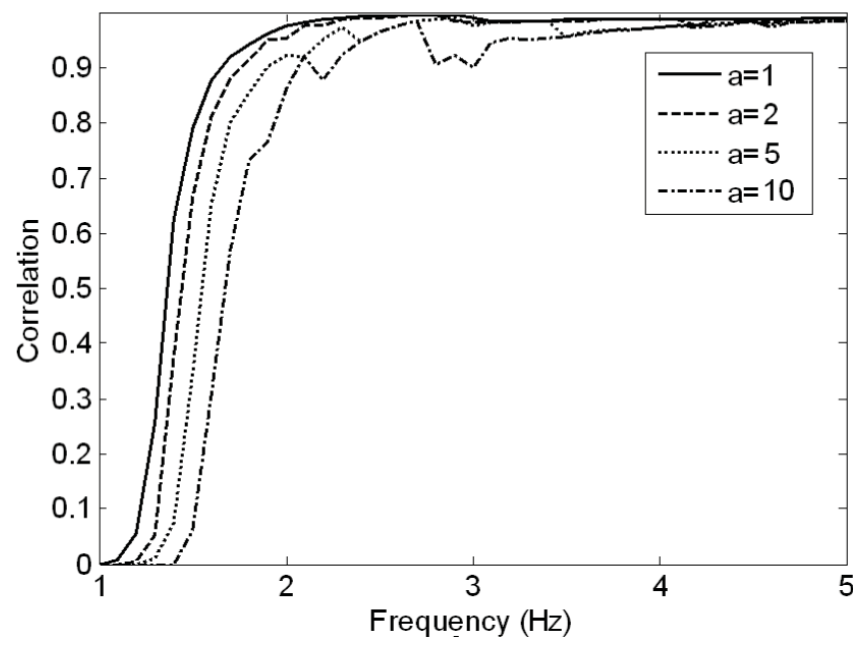

Fig. 6. EMD success in removing interference. Resemblance (correlation) of the recovered signal to the principal signal versus interference signal frequency for amplitude ratios of $1-10$.

diagram of a quadrature mono-static radar sensor used for these measurements is shown in Fig. 1. An HP83640B signal generator operating at $2.4 \mathrm{GHz}$ was used as the signal source. RPS-2-30 splitters, ZFM4212 mixers from Mini-circuits and a Narda 4923 circulator were used. A subject was seated at a distance of 1.5 meters and the transmit $\mathrm{CW}$ power at the antenna input was $0 \mathrm{dBm}$. An ASPPT2988 antenna was used with $8 \mathrm{dBi}$ gain and 60 degrees beam-width. The I and Q mixers' outputs are low pass filtered for anti-aliasing, amplified using a SR560 LNA (cutoff $100 \mathrm{~Hz}, 40 \mathrm{~dB}$ gain), and recorded by a NI USB6259 16 bit data acquisition device to the PC at a sampling rate of $1 \mathrm{kHz}$. A chest band (Pneumotrace 1132 piezoelectric respiration transducer) was used as a respiratory signal reference, and an UFI 1010 finger pulse sensor was used as a heart signal reference. An ADXL203 dual axis accelerometer chip was attached to the antenna. The antenna was placed on a fixture that was shaken mechanically during parts of the measurement. The mechanical shake was produced using a fan that was placed on the antenna fixture and switched on to create vibrations with the frequency spectrum of 2.5-6 Hz. The motion artifact signals were periodic but not sinusoidal.

Three datasets with varying amplitude ratios were examined in this section. The output data was processed using a Matlab code that performs linear demodulation [1] and comparison with the reference heart signal. Fig. 7 shows signals received from sensor (I-Q channels), heart signal from reference finger pulse sensor and motion signals measured by the accelerometer, for Dataset 1. This Dataset has the heart frequency of about $1 \mathrm{~Hz}$ and amplitude ratio of 3 . As it can be seen transmitter antenna starts shaking at $\mathrm{t}=60 \mathrm{~s}$. The effect of antenna's motion artifact can be seen in sensor's output in Fig. 7 starting at $\mathrm{t}=60 \mathrm{~s}$. Since in a Doppler radar sensor the measured quantity is the radial motion of the subject, the most important motion artifact component will be along the $x$-axis (Fig. 1).

The sensor signal has several components: a large respiration component, a small heart component, and a motion artifact component. Small amplitude of heart signal compared

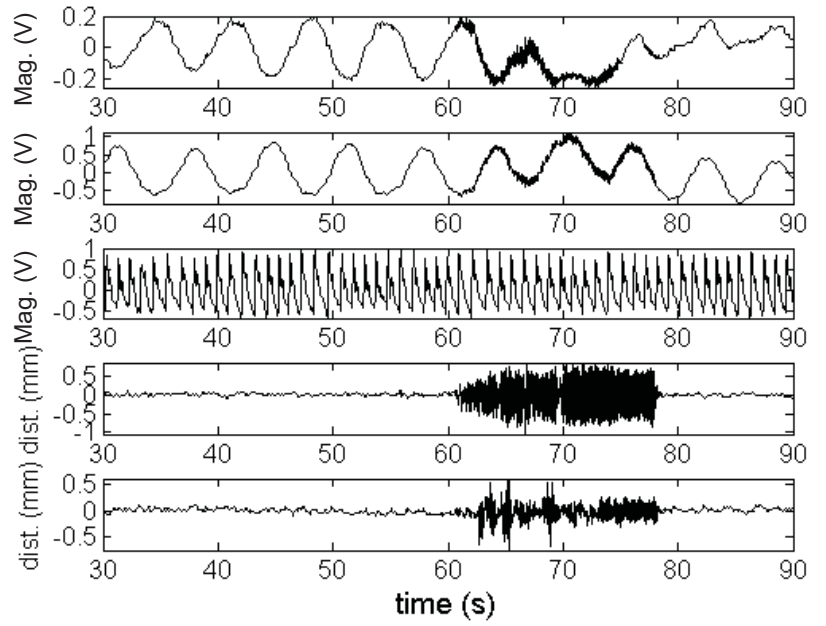

Fig. 7. Signals recorded in the experiment. From the top: Radar sensor $\mathrm{I}$, and $\mathrm{Q}$, finger pulse sensor as reference and $\mathrm{X}$ and $\mathrm{Y}$ shake signals for Dataset 1 .
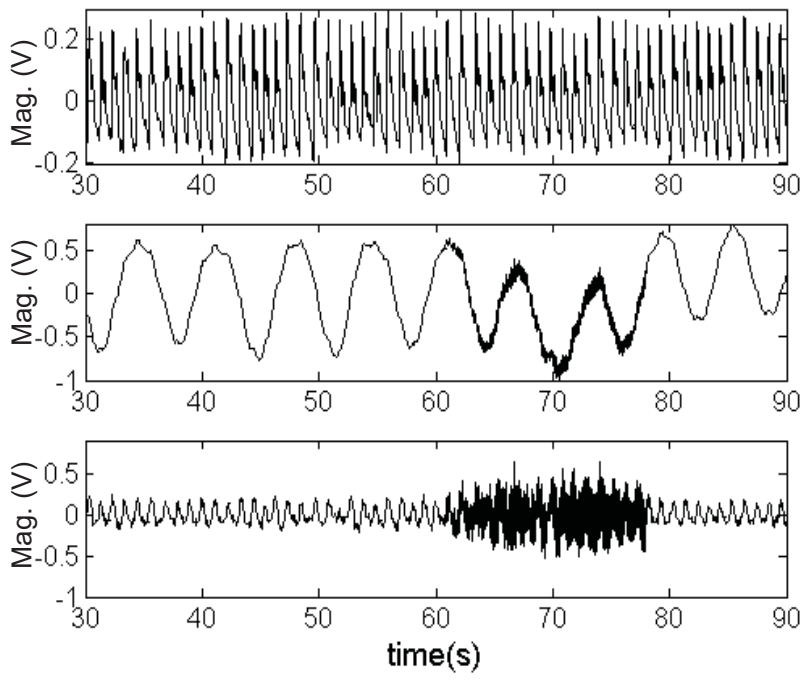

Fig. 8. Signals for Dataset 1. From top to bottom: reference heart signal, demodulated sensor signal (I-Q combined) and high-pass filtered sensor signal.

to that of respiration makes it difficult for EMD to properly decompose sensor's output into corresponding IMFs. As a solution, respiration trace can be attenuated using a high pass filter, as shown in Fig. 8 with the reference heart signal. This signal is then decomposed into IMFs and as outlined in Section II, its IMFs are calculated and plotted in Fig. 9.

By visually inspecting the IMFs and comparing them to the motion artifact signal recorded with accelerometers (shown in Fig. 7) it is clear that the first IMF of the sensor signal is very similar to the antenna motion signal. As a quantitative measure, cross-correlation of each of the IMFs and the signal from the accelerometer have been calculated and shown in Table I using methods described in [11]. The first IMF shows major correlation with $\mathrm{X}$ and $\mathrm{Y}$ motion artifact signals obtained from the accelerometer while other IMFs have almost no correlation with the motion artifact signals. Knowing that the motion artifact signal appears in the first IMF it can easily 

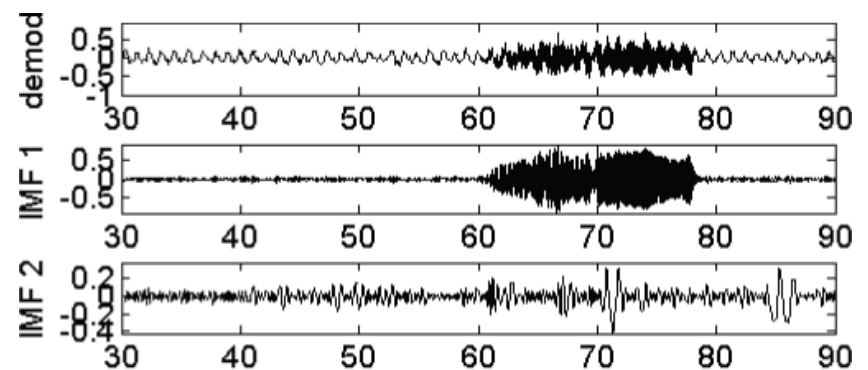

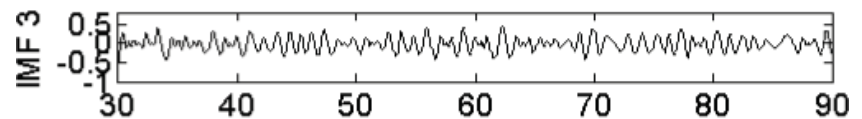

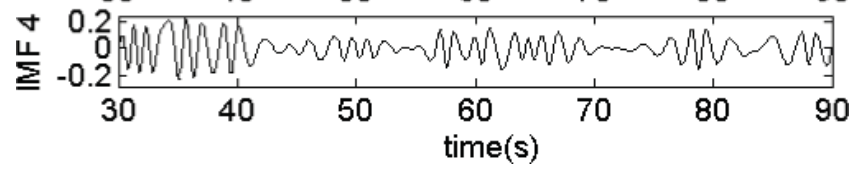

Fig. 9. High-pass filtered linear demodulated sensor signal and four of its first IMFs for Dataset 1.

TABLE I

Cross Correlation of Each of the IMFs AND the Shake Signals FOR DATASET 1 (FROM AXES X AND Y)

\begin{tabular}{|l|l|l|}
\hline IMF \# & Cross Corr X & Cross Corr Y \\
\hline IMF 1 & -72.85 & -18.18 \\
\hline IMF 2 & -1.00 & -0.24 \\
\hline IMF 3 & -0.44 & 0.31 \\
\hline IMF 4 & 0.13 & 0.07 \\
\hline IMF 5 & 0.03 & -0.11 \\
\hline IMF 6 & 0.01 & 0.59 \\
\hline IMF 7 & 0.07 & 0.05 \\
\hline IMF 8 & -0.47 & -1.24 \\
\hline
\end{tabular}
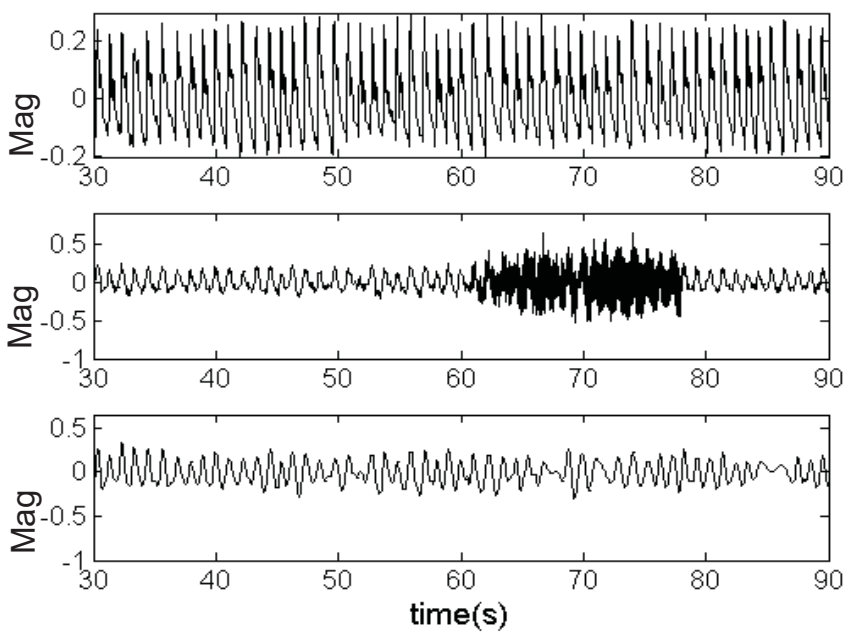

Fig. 10. Reference finger pulse (top), demodulated high-pass filtered sensor signal (middle) and motion cancelled signal for Dataset 1 (bottom).

be removed by summing up IMF 2-8. This will give the reconstructed sensor signal which is shown in Fig. 10. It can be seen that the motion artifact cancelled signal follows the reference heart signal very well in time domain.

As a final measure of accuracy and effectiveness of the method, the motion artifact cancelled signal is passed through

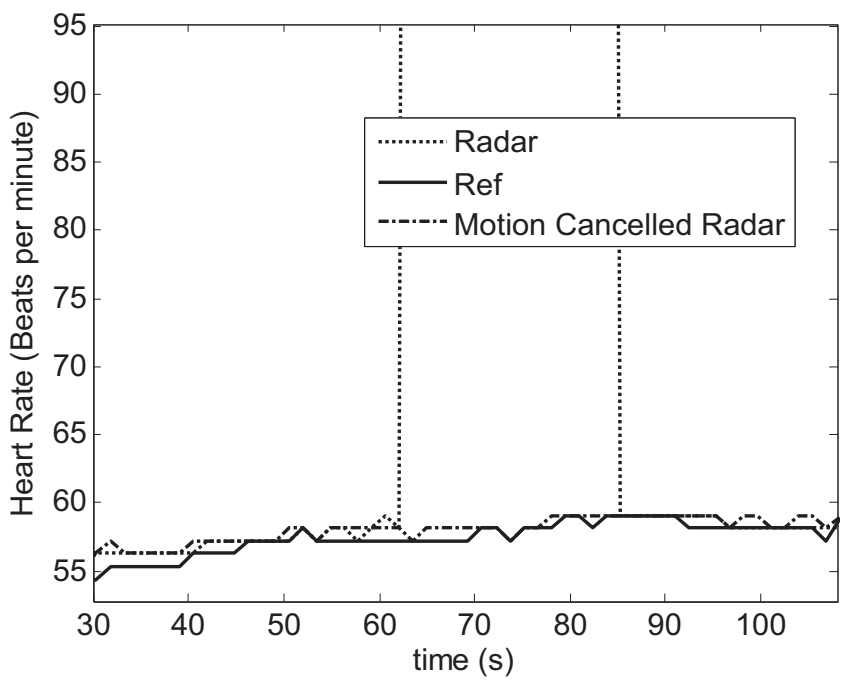

Fig. 11. Calculated heart rate of the subject from reference, radar sensor, and motion cancelled radar sensor signals obtained from Dataset 1.
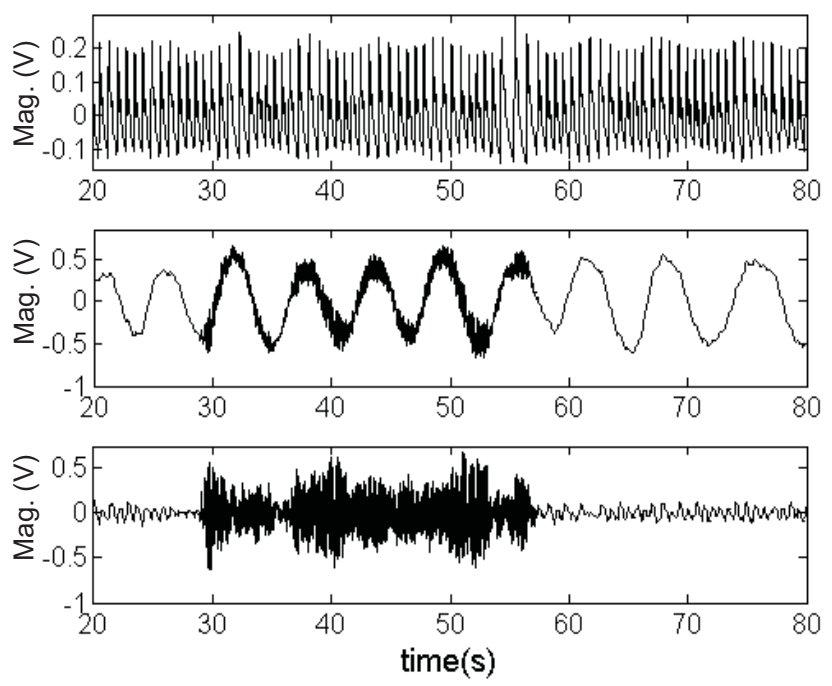

Fig. 12. Reference finger pulse (top), demodulated radar (middle), and highpass filtered radar (bottom) signals for Dataset 2.

the heart rate estimator for calculating heart rate [11]. Fig. 11 shows the extracted heart rate with and without motion cancellation compared to the wired reference. It can be seen that the motion cancelled signal tracks the reference finger pulse with high accuracy even in presence of the mechanical shake (from $t=60 \mathrm{~s}$ to $\mathrm{t}=80 \mathrm{~s}$ ), which proves the effectiveness of the proposed method. Heart rate estimated from the motioncancelled sensor signals has root mean square error (RMSE) of 0.6 beats per minute (BPM). Using the method described in [11], it was also possible to extract heart rate for this dataset, although with slightly lower accuracy of 1.2 BPM.

This method has also been applied to two other datasets with interference to heart signal amplitude ratios of 8 and 13 respectively. In Dataset 2, amplitude ratio was 8 and subject heart rate was $1.4 \mathrm{~Hz}$, resulting in slightly lower frequency separation. Fig. 12 shows the reference finger pulse (top), demodulated radar (middle), and high pass filtered radar (bottom) signals 


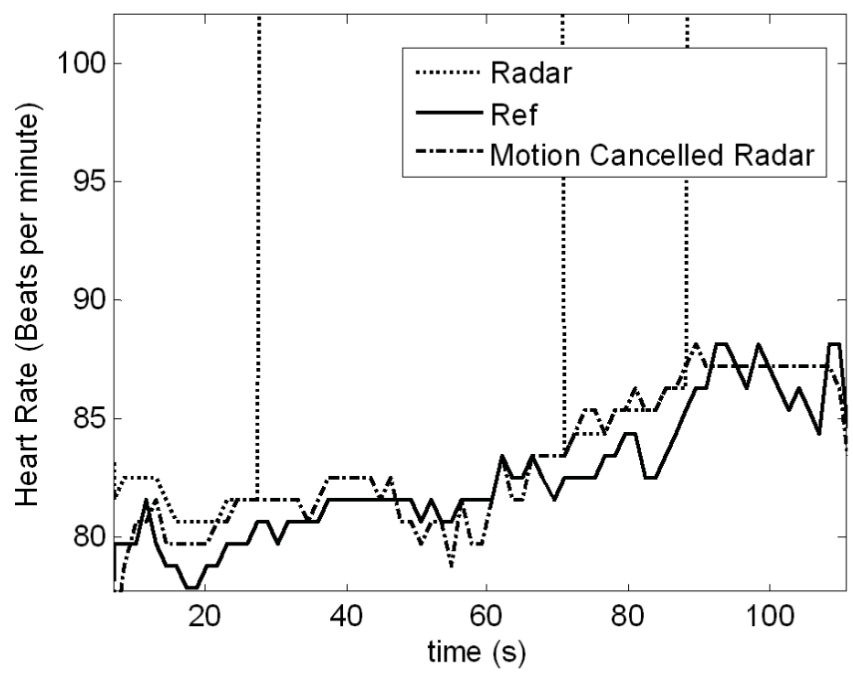

Fig. 13. Calculated heart rate for heart reference, radar sensor, and motion cancelled radar sensor data for Dataset 2.
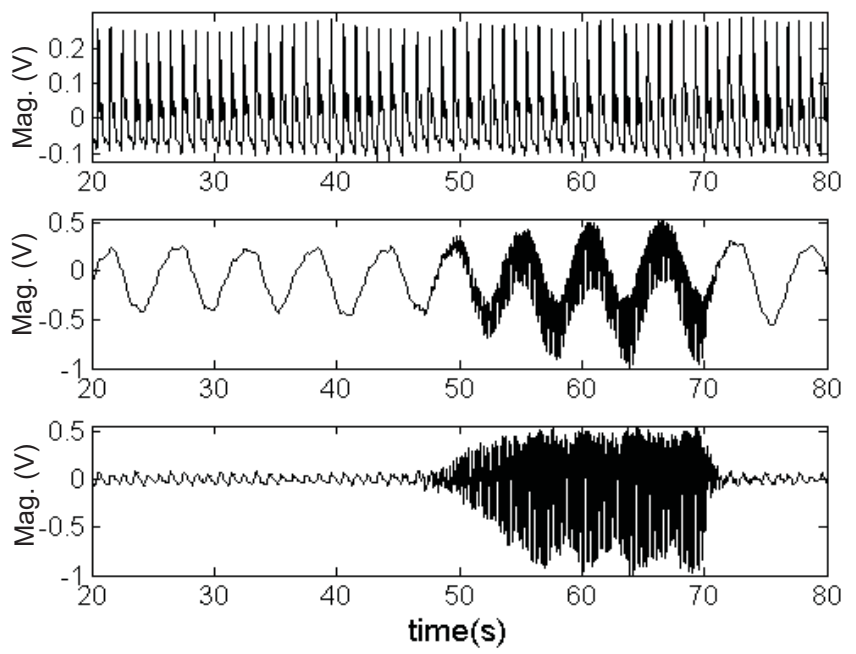

Fig. 14. Reference finger pulse (top), demodulated radar (middle), and highpass filtered radar (bottom) signals for Dataset 3.

for Dataset 2. Fig. 13 shows calculated heart rate with and without motion cancellation compared to thewired reference, demonstrating that the proposed method is working effectively. In this case, heart rate RMSE of 1 BPM was slightly higher than for Dataset 1, likely due to lower frequency separation, as predicted by simulation results. Method [11] fails in this case. Dataset 3 was taken with the same subject as Dataset 1, with the amplitude ratio of twelve. Fig. 14 shows the reference finger pulse (top), demodulated radar (middle) and high pass filtered radar (bottom) signals for Dataset 3.

Fig. 15 shows calculated heart rate with and without motion cancellation compared to the wired reference, for Dataset 3. In this case, even though the amplitude ratio was significantly larger, heart rate was extracted with RMSE of 0.6 BPM, same as for Dataset 1 . This is consistent with the simulation results shown in Fig. 5 and Fig. 6, indicating that increasing amplitude ratio has little effect on the separation success for sufficient separation frequency. The method in [11] fails for

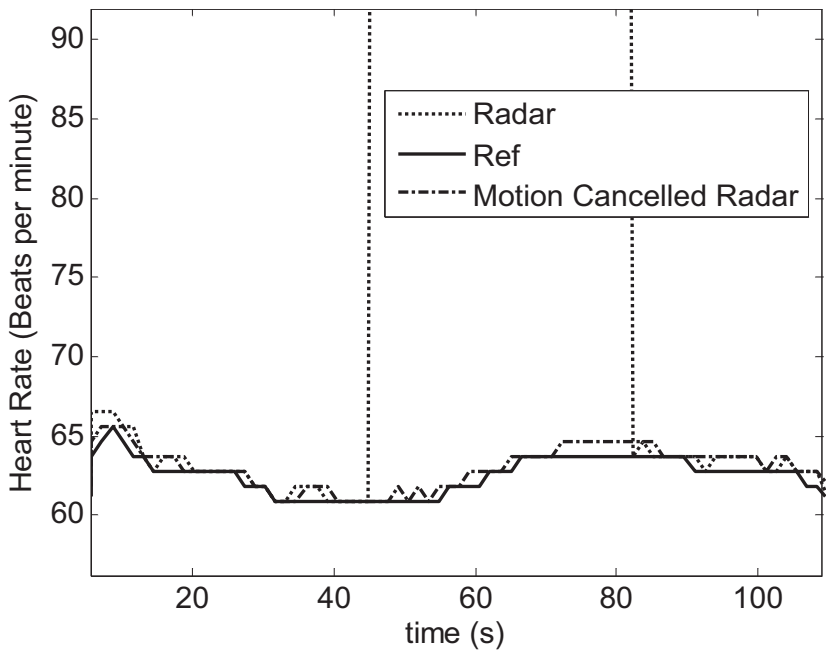

Fig. 15. Calculated heart rate for heart reference, radar sensor, and motion cancelled radar sensor data for Dataset 3.

larger interference due to inherent susceptibility to noise when two signals are subtracted. Due to the fact that heart and motion artifact spectra are not known beforehand, and that these two spectra may have a small frequency separation, it is difficult to use fixed filters for interference cancellation. Even if the spectra of the two signals were known, if the frequency separation is of same order as heart frequency, filtering interference would result in filtering higher harmonics of the heart signals, thus resulting in a lower heart rate estimation accuracy [14].

\section{CONCLUSION}

Doppler radar sensing has shown promise for subject detection and physiological monitoring. However, the mechanical motion of the sensor itself can severely limit the usefulness of this approach. In this paper, we have employed Empirical Mode Decomposition to isolate and cancel the interference signal. Theoretical analysis and simulation results have illustrated the potential of this technique for a wide range of frequency separation and amplitude ratios of physiological signals and motion artifacts. Experimental results from three different datasets confirm the effectiveness of this method. Motion cancellation was performed with sufficient precision to extract the heart rate with accuracy within 1 beat per minute, even in the presence of motion artifacts an order of magnitude larger than the heart signal. This method can be further applied to separate other sources of interference from human physiological signals, such as background clutter motion, and undesired subject motion.

\section{REFERENCES}

[1] W. Massagram, V. M. Lubecke, A. Host-Madsten, and O. BoricLubecke, "Assessment of heart rate variability and respiratory sinus arrythmia via doppler radar," IEEE Trans. Microw. Theory Tech., vol. 57, no. 10, pp. 2542-2549, Oct. 2009.

[2] C. Huey-Ru, K. Hsin-Chih, L. Fu-Ling, H. Tzuen-His, K. Chi-Shin, and O. Ya-Wen, "60-GHz millimeter-wave life detection system (MLDS) for noncontact human vital-signal monitoring," IEEE Sensors J., vol. 12, no. 3, pp. 602-609, Mar. 2012. 
[3] D. Girbau, A. Lazaro, A. Ramos, and R. Villarino, "Remote sensing of vital signs using a doppler radar and diversity to overcome null detection," IEEE Sensors J., vol. 12, no. 3, pp. 512-518, Mar. 2012.

[4] M. Zakrzewski, H. Raittinen, and J. Vanhala, "Comparison of center estimation algorithms for heart and respiration monitoring with microwave doppler radar," IEEE Sensors J., vol. 12, no. 3, pp. 627-634, Mar. 2012.

[5] Y. Xiao, C. Li, and J. Lin, "A portable noncontact heartbeat and respiration monitoring system using 5-GHz radar," IEEE Sensors J., vol. 7, no. 7, pp. 1042-1043, Jul. 2007.

[6] C. Li and J. Lin, "Random body movement cancellation in doppler radar vital sign detection," IEEE Trans. Microw. Theory Tech., vol. 56, no. 12, pp. 3143-3152, Dec. 2008.

[7] Q. R. Razligh and N. Kehtarnavaz, "Image blur reduction for cell-phone cameras via adaptive tonal correction," in Proc. IEEE Int. Conf. Image Process. vol. 1. San Antonio, TX, Sep. 2007, pp. 113-116.

[8] E. F. Greneker and D. J. Zywicki, "Stabilizing motion in a radar detection system using ultrasound radar range information," U.S. Patent 6909397, Jun. 21, 2005.

[9] A. Singh and V. M. Lubecke, "Respiratory monitoring and clutter rejection using a CW doppler radar with passive RF tags," IEEE Sensors J., vol. 12, no. 3, pp. 558-565, Mar. 2012.

[10] I. Mostafanezhad, B. K. Park, O. Boric-Lubecke, and V. L. A. HostMadsen, "Sensor nodes for doppler radar measurements of life signs," in IEEE MTT-S Int. Microw. Symp. Dig., vol. 1. Honolulu, HI, Jun. 2007, pp. 1241-1244.

[11] I. Mostafanezhad, O. Boric-Lubecke, V. Lubecke, and A. Host-Madsen, "Cancellation of unwanted motion in a handheld doppler radar used for life sign monitoring," in IEEE MTT-S Int. Microw. Symp. Dig., Atlanta, GA, Jun. 2008, pp. 1171-1174.

[12] N. E. Huang, Z. Shen, S. R. Long, M. C. Wu, H. H. Shih, Q. Zheng, N.-C. Yen, C. C. Tung, and H. H. Liu, "The empirical mode decomposition

and the Hilbert spectrum from nonlinear and non-stationary time series analysis," Proc. Royal Soc. Lond. A, vol. 454, no. 1971, pp. 903-995, 1998.

[13] L. Rasolofondraibe, B. Pottier, P. Marconnet, and X. Chiementin, "Capacitive sensor device for measuring loads on bearings," IEEE Sensors J., vol. 12, no. 6, pp. 2186-2191, Jun. 2012.

[14] D. Mandic, M. Golz, A. Kuh, D. Obradovic, and T. Tanaka, Signal Processing Techniques for Knowledge Extraction and Information Fusion. New York: Springer-Verlag, 2008.

[15] I. Mostafanezhad, O. Boric-Lubecke, V. Lubecke, and D. Mandic, "Application of empirical mode decomposition in removing fidgeting interference in doppler radar life signs monitoring devices," in Proc. Annu. Int. Conf. IEEE Eng. Med. Biol., Minneapolis, MN, Sep. 2009, pp. 340-343.

[16] O. A. Omitaomu, V. A. Protopopescu, and A. R. Ganguly, "Empirical mode decomposition technique with conditional mutual information for denoising operational sensor data," IEEE Sensors J., vol. 11, no. 10, pp. 2565-2575, Oct. 2011.

[17] Empirical Mode Decomposition. (2007) [Online]. Available: http://perso.ens-lyon.fr/patrick.flandrin/emd.html

[18] G. Rilling and P. Flandrin, "One or two frequencies? The empirical model decomposition answers," IEEE Trans. Signal Process., vol. 56, no. 1, pp. 85-95, Jan. 2007.

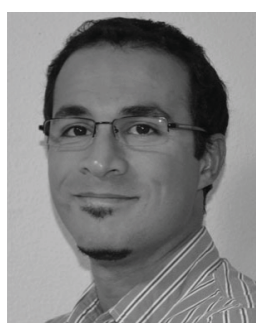

Isar Mostafanezhad (S'06-M'10) received the Ph.D. degree in electrical engineering from the University of Hawaii at Manoa, Honolulu, in 2010.

$\mathrm{He}$ is currently with the University of Hawaii at Manoa, where he is involved in research on reducing the noise and interference in wireless vital signs monitoring devices. He implemented hardware and also used signal processing techniques to improve the range and accuracy of the vital signs monitoring sensor. He was with Kai Medical, Honolulu, where he was engaged on commercializing the contactless respiration monitor and passing it through regulatory bodies and the FDA. His current research interests include RF, microwaves, biomedical signal processing, and sensing of vital signs.

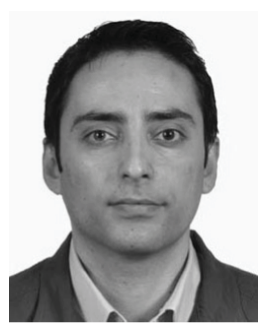

Ehsan Yavari (S'11) received the B.S.E.E. degree from the Ferdowsi University of Mashhad, Mashhad, Iran, and the M.Sc. degree in electronics from Tarbiat Modares University, Tehran, Iran, in 2003 and 2006, respectively. He is currently pursuing the $\mathrm{Ph} . \mathrm{D}$. degree at the Electrical Engineering Department, University of Hawaii at Manoa, Honolulu.

His current research interests include applications of wireless technologies in health monitoring and sensing.

Mr. Yavari is a member of the IEEE Microwave Theory and Technologies Society and the IEEE Engineering in Medicine and Biology.

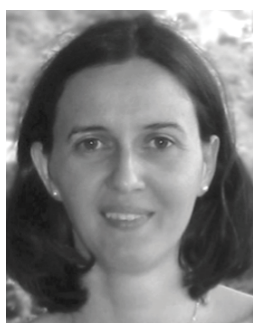

Olga Boric-Lubecke (S'90-M'90-SM'01) received the B.Sc. degree from the University of Belgrade, Belgrade, Yugoslavia, the M.S. degree from the California Institute of Technology, Pasadena, and the $\mathrm{Ph} . \mathrm{D}$. degree from the University of California at Los Angeles, Los Angeles, in 1989, 1990, and 1995, respectively, all in electrical engineering.

She has been with the University of Hawaii at Manoa, since 2003, where she is currently a Professor of electrical engineering. She was prevously with Bell Laboratories, Lucent Technologies, Murray Hill, NJ, where she was involved in research on RF integrated circuit technology and biomedical applications of wireless systems. From 1996 to 1998, she was with the Institute of Physical and Chemical Research, Sendai, Japan, 1995 to 1996, she was with the Jet Propulsion Laboratory, NASA, Pasadena, CA. She has authored or co-authored over 150 papers in journals and conferences. Her research papers are featured in various newspapers, magazines, and radio programs. Her current research interests include silicon RF integrated circuits, high-frequency integrated circuits, biomedical applications, and renewable energy.

Dr. Boric-Lubecke is the adviser-author of several award winning IEEE MTT-S and EMB-S student papers, and serves regularly on MTT-S committees. She is currently an Associate Editor of the IEEE Microwave and Wireless Components Letters, and the IEEE EMBC. She is the Co-Founder and was the Chief Technical Advisor of Kai Medical, a start-up company.

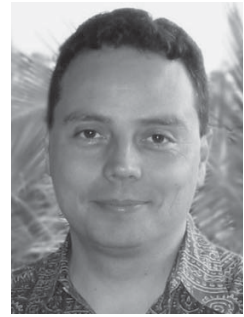

Victor M. Lubecke (S'86-M'86-SM'98) received the B.S.E.E. degree from the California Polytechnic Institute, Pomona, in 1986, and the M.S. and Ph.D. degrees in electrical engineering from the California Institute of Technology, Pasadena, in 1990 and 1995, respectively.

$\mathrm{He}$ is currently a Professor of electrical engineering with the University of Hawaii at Manoa, Honolulu, where he has been since 2003. He was with Bell Laboratories, Lucent Technologies, where he was involved in research on sensing and monitoring technologies for biomedical and industrial applications and on microelectromechanical systems (MEMS) and 3-D wafer-scale integration technologies for wireless and optical communications. From 1987 to 1996, he was with Jet Propulsion Laboratory, National Aeronautics and Space Administration, and from 1996 to 1998, he was with the Institute for Physical and Chemical Research, Sendai, Japan, where he was involved in research on remote sensing and space communications applications and related MEMS and terahertz technologies. He has authored or co-authored over 150 peer-reviewed papers in journals and conferences. He holds 6 U.S. patents with several pending. His current research interests include remote sensing and imaging technologies, biomedical sensors, animal tracking and monitoring, MEMS, heterogeneous integration, and microwave/terahertz radios.

Dr. Lubecke was a co-recipient of the Emerging Technology Award at TechConnect 2007. He was the Co-Founder and the CTO of Kai Medical, Honolulu, a start-up company. 


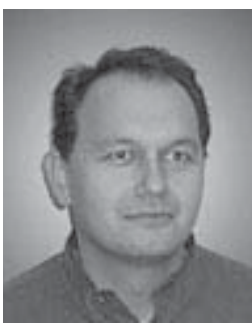

Danilo P. Mandic (F'13) is currently a Professor of signal processing with Imperial College London, London, U.K., where he is involved in research on nonlinear and multivariate adaptive signal processing and spectral estimation. $\mathrm{He}$ is a Guest Professor with KU Leuven, Belgium, a Frontier Researcher with RIKEN Japan. He has authored or co-authored two research monographs entitled Recurrent Neural Networks for Prediction (Wiley, 2001) and Complex Valued Nonlinear Adaptive Filters (Wiley, 2009).

Prof. Mandic is a member of the IEEE Technical Committee on Signal Processing Theory and Methods, and an Associate Editor of the IEEE Signal Processing Magazine, the IEEE TRANSACTIONS on Signal Processing, and the IEEE Transactions on Neural NETWORKS. 